\title{
Mean Flow and Turbulence Measurements in the Near Field of a Fighter Aircraft Wing-Tip Vortex at High Reynolds Number and Transonic Flow Conditions
}

\author{
Fenella de Souza and Ben H.K. Lee \\ Institute for Aerospace Research \\ National Research Council Canada \\ Ottawa, Ontario, Canada K1A 0R6 \\ e-mail: fenella.desouza@nrc.ca, ben.lee@nrc.ca
}

\begin{abstract}
Mean and turbulent flow characteristics of the wing-tip vortex generated by a $6 \%$ scale model of the F/A-18 fighter aircraft equipped with AIM-9 sidewinders were investigated experimentally. This particular configuration was studied as part of the NRC/IAR stores clearance and certification program. However, the present study focuses on the near field structure of the tip vortex, as the existing literature contains relatively little information on tip vortices generated at such high Reynolds numbers $\left(4.4 \times 10^{6}\right.$, based on wing chord), and even less on the tip vortices of fighter aircraft. The experiments were conducted in the transonic test section of the NRC/IAR $1.5 \mathrm{~m} \times 1.5 \mathrm{~m}$ trisonic blowdown wind tunnel. The present paper documents results obtained in a single cross-flow plane located immediately downstream of the wing trailing edge, for two angles of attack, $4.5^{\circ}$ and $13^{\circ}$, and at a single freestream Mach number of 0.75. Mean velocities and flow angularities were measured using a five-hole directional velocity probe as well as an x-array constant temperature hot-wire probe. The latter also provided estimates of the turbulence intensities, Reynolds stresses and turbulent kinetic energy in the measurement plane. The results shed some light on the fairly complex interaction, particularly at the higher angle of attack, of the wing-tip vortex and the wake of the AIM-9 tip missile.
\end{abstract}

\section{Introduction}

There has been renewed interest in studying the formation, structure and development of aircraft wing-tip vortices. These trailing vortices can be a serious hazard for smaller, following aircraft. Although many recent studies have been devoted to this topic, most have used generic rectangular wings with symmetrical profiles (eg. Devenport et al. ${ }^{1,2}$, Chow et $a l^{3}$ ), and relatively few have been performed at high enough Reynolds numbers to reflect realistic flight conditions.

The present results were obtained from a wind tunnel investigation of the flowfield around the wing of an F/A-18 fighter aircraft, using a five-hole directional velocity probe as well as hot-wire anemometry. A model F/A-18 was tested in a clean wing configuration (ie. without any wing stores or pylons installed) except for AIM-9 wing tip missiles which were mounted for all the tests. This configuration was studied not from the point of view of safety for following aircraft, but to provide flowfield measurements for computing the trajectories of light weight stores ejected from the aircraft. However, the present paper focuses on the measurements obtained in the near field of the wing-tip vortex. Tip vortices of fighter aircraft have not often been studied in the past. Also, relatively few measurements of trailing vortices have been acquired at such high Reynolds and Mach numbers.

\section{Experimental Facilities and Model}

The experiments were conducted in the transonic test section of the NRC/IAR $1.5 \mathrm{~m} \times 1.5 \mathrm{~m}$ trisonic blowdown wind tunnel. This facility has a Mach number range of 0.1 to 4.2. At subsonic and transonic flow conditions, a computerized control system sets the test section Mach number to within \pm 0.003 of the desired value over a wide range of test conditions. The stagnation pressure can be maintained constant to 
within $\pm 0.14 \mathrm{kPa}( \pm 0.02 \mathrm{psia})$ throughout a wind tunnel run. The test section walls are perforated with $1.27 \mathrm{~cm}$ diameter holes inclined at $30^{\circ}$ to the flow direction in order to minimize wall interference. The wall porosity is adjustable and was set at $4 \%$ for the present measurements. This facility has been described in more detail by Ohman et al. ${ }^{4}$ and Ohman and Brown ${ }^{5}$.

The wing-tip vortex was generated by a rigid $6 \%$ scale wind tunnel model of the F/A-18 fighter aircraft. The model was supported by a roof-mounted sting (Figure 1). The mounting bracket permitted variation of the model angle of attack in discrete steps. During the present series of experiments, results were obtained for two moderate angles of attack: $4.5^{\circ}$ and $13^{\circ}$.

The model construction involved the assembly of three major components: a stainless steel central section with a one piece wing box, an aluminium alloy forebody with integral leading edge extensions (LEX) and a stainless steel rear fuselage. These components were assembled through bolted and spigotted joints. Through-flow air inlets were fitted without using any internal chokes (ie. fully open). Vertical fins were bolted to the rear fuselage. Due to mechanical interference with the articulated sting used to support the measurement probe, the horizontal stabilators were removed. However, since all measurements were well upstream of the location of the stabilators, this was not expected to have any effect on the results. Boundary layer transition strips were placed on the wings, LEX, vertical fins and forebody of the model.

Due to the particular wing geometry, the flow structure in the vicinity of the wing-tip can be expected to be considerably more complex than that typically investigated in tip vortex studies using rectangular halfwings of symmetrical profile. AIM-9 sidewinders were mounted on the wing-tips as shown in Figure 2, and thus the resulting flow was due to the interaction of the wing-tip vortex, the wake of the main body of the missile (similar to an ogive cylinder), and the additional vortical flow generated by the canards and fins of the AIM-9. The underwing of the model was clean (ie. no stores or pylons were installed). Wing flaps were bolted to the wing box and set to zero deflection.

The model coordinate system is shown in Figure 3. The $x, y$ and $z$ coordinates are measured in the streamwise, vertical (upwards) and lateral (towards the port side of the model) directions, respectively. The origin of the coordinate system is located in the vertical mid-plane of the fuselage, $92.2 \mathrm{~mm}(3.63$ inches) ahead of the model forebody (along the fuselage centreline) and $152.4 \mathrm{~mm}$ (6.00 inches) below (and along a line perpendicular to) the fuselage centerline. The present study documents results obtained in the cross-flow plane located at a single axial measurement station, $87.6 \mathrm{~cm}(4.2 \mathrm{c}$, where $c$ is the model wing mean aerodynamic chord) downstream of the origin, as shown in the figure.

\section{Instrumentation and Data Acquisition}

An articulated sting with accurate optical tracking was used to position the measurement probes at the desired locations in the flowfield. The mean velocities and flow angularities in the near field of the tip vortex were measured using a five-hole directional velocity probe. These quantities were also obtained, along with turbulence intensities and the Reynolds stresses, via hot-wire anemometry using single-, parallel- and X-wire probes. Measurements were acquired at freestream Mach numbers of 0.75 and 0.9 , and at a single Reynolds number of $4.4 \times 10^{6}$ (based on the model wing mean aerodynamic chord of $21 \mathrm{~cm}$ ). Due to the large amount of data collected, only the results obtained at Mach 0.75 are presented in this paper. The measurement probes and techniques are described in detail below.

\section{Five-Hole Probe}

The five-hole probe head consisted of a $4.06 \mathrm{~mm}$ OD cap with five accurately positioned holes for slide fitting $0.81 \mathrm{~mm}$ OD, $0.51 \mathrm{~mm}$ ID hypodermic pressure tubing (Figure 4). This tubing was contained in a $3.4 \mathrm{~mm}$ OD tube which in turn fit inside a $6.35 \mathrm{~mm}$ OD tube to comprise the main body of the probe. With the five pressure tubes epoxied into place, the cap was machined to form a four-sided pyramid with faces at $45^{\circ}$ to the probe axis. The probe "static" pressures (taken from the four peripheral holes) were measured 
by individual $34.5 \mathrm{kPa}$ (5 psi) differential transducers, with the probe "total" pressure (taken from the central hole) taken as reference and measured by a $345 \mathrm{kPa}(50 \mathrm{psi})$ absolute transducer.

The flow velocity and angularity were deduced from the probe pressure coefficients using calibration lookup tables, which had been established previously for a number of probes of identical design and construction. These probes were calibrated in a rake assembly with respect to flow angles, Mach number and total pressure in the Lockheed Compressible Flow Wind Tunnel at Mach numbers of 0.6, 0.7 and 0.8 (see Ohman ${ }^{6}$ ). To verify the asymmetry and sensitivity of the probe used in this study, a number of calibration runs were performed, and the maximum uncertainties in the derived data were estimated to be $\pm 0.1^{\circ}$ for the flow pitch and yaw angles, \pm 0.0048 for the normalized (with respect to freestream velocity) axial flow velocity, \pm 0.0017 for the normalized cross-flow velocities and \pm 0.006 for the Mach number?

The five-hole probe measurements were acquired at a single model angle of attack of $4.5^{\circ}$. During a typical wind tunnel run, the five-hole probe would traverse axially for a distance of $40 \mathrm{~cm}$ at a speed of $3 \mathrm{~cm} / \mathrm{s}$ starting from the fully retracted position of the articulated sting. The probe would then be positioned to another preprogrammed vertical station and returned at the same speed to its fully retracted position. Only the measurements obtained at $x / c=4.2$ are presented in this study. The measurement grid for this cross-flow plane is shown in Figure 5.

The position of the five-hole probe relative to the parent aircraft was measured non-intrusively and in real time by an optical tracking system, OPTOTRAK/3020. Markers (IREDs) were embedded on the parent aircraft, the probe holder and on the wind tunnel wall. Two OPTOTRAK cameras, each of which consisted of two one-dimensional sensors (CCD arrays), were installed in the plenum chamber of the wind tunnel as shown in Figure 1. The maximum uncertainties in the measured probe position using this system were $\pm 0.25 \mathrm{~mm}$ in the axial ( $x$ ) and lateral ( $z$ ) directions, $\pm 0.125 \mathrm{~mm}$ in the vertical ( $y$ ) direction, $\pm 0.25^{\circ}$ in pitch and yaw angles and $\pm 1^{\circ}$ in roll angle ${ }^{7}$.

\section{Hot-Wire Anemometry}

The Dantec Streamline constant temperature anemometry system was used for the turbulence measurements. Measurements were acquired with a model 55P11 single-wire probe, a model 55P71 parallel-wire probe and a model 55P61 x-wire probe. Platinum-plated tungsten wires of 7.6 microns in diameter and approximately $1.25 \mathrm{~mm}$ in length were used for all probes. The single- and $\mathrm{x}$-wires were operated at a resistance overheat ratio of 1.8. The two wires of the parallel-wire probe were operated at two different overheat ratios, 1.5 and 1.8 , in order that their sensitivities to density and velocity be sufficiently different to permit decoupling of the fluctuations of these two quantities.

All hot-wire probes were calibrated with respect to both velocity and density over the range of expected test conditions. Calibration was performed in the NRC/IAR 5" x 5" pilot wind tunnel, which is a 1:12 scaled prototype model of the NRC/IAR $1.5 \mathrm{~m} \times 1.5 \mathrm{~m}$ trisonic blowdown tunnel. The calibration typically covered a range of Mach numbers from 0.66 to 0.8 , and a range of densities from 1.1 to $1.7 \mathrm{~kg} / \mathrm{m}^{3}$. The temperature remained constant during calibration. $\mathrm{X}$-wire probes were also calibrated for directional sensitivity, using the Dantec Streamline calibration jet facility. The probes were pitched through a range of flow angles from $-20^{\circ}$ to $+20^{\circ}$ for a number of constant velocity levels, representative of the range of expected test conditions. From this calibration, average pitch factors were determined for each wire.

The present paper focuses specifically on the turbulence measurements acquired with the $\mathrm{x}$-wire probe. The single- and parallel-wire probe served mainly to verify the accuracy and repeatability of the streamwise velocity measurements, which were found to be acceptable at the present time. The x-wire measurements represent a single cross-flow plane located very close to the port-side wing trailing edge $(x / c=4.2)$ at the two different model angles of attack $\left(4.5^{\circ}\right.$ and $\left.13^{\circ}\right)$. The measurement grid in this cross-flow plane is shown in Figure 5. During a typical wind tunnel run, the hot-wire probe was positioned at a single lateral station, and was traversed across three to six preprogrammed vertical stations. At each vertical station, 
between 4 and 8 seconds of data were acquired at a sampling rate of $30 \mathrm{kHz}$ per wire. The position of the $\mathrm{x}$-wire probe relative to the parent aircraft was measured by the optical tracking system in the same manner as that described for the five-hole probe measurements.

\section{Other Instrumentation}

A thermocouple was mounted on the probe holder so as to measure the temperature as close as possible to the hot-wire sensor. These measurements were used to correct the hot-wire signals for flow temperature variation. Total temperature variation in the wind tunnel was inferior to $3 \mathrm{~K}$ during a typical run.

\section{Mean Velocity Distributions in the Plane $x / c=4.2$}

Figure 6 displays the normalized cross-flow velocity vectors in the cross-flow plane located immediately behind the wing trailing edge $(x / c=4.2)$, as measured with the five-hole probe at $M=0.75$ and $\alpha=4.5^{\circ}$. For the $\mathrm{x}$-wire survey, the measurement grid was refined in the region surrounding the trailing edge (see Figure 5). The corresponding results are shown in Figure 7, and are qualitatively similar to those obtained with the five-hole probe. The five-hole probe measurements of the mean velocity components are estimated to be more accurate than those from the $\mathrm{x}$-wire; differences between the two figures are due mainly to uncertainties in the mean velocities measured by the $x$-wire, which are estimated to be less than $5 \%$ for the streamwise component, and up to $10 \%$ for the transverse components. Figure 6 demonstrates that the wing-tip vortex is well developed as the flow leaves the wing trailing edge, with tangential velocities of the order of 5\% of the freestream velocity. The results obtained at $\alpha=13^{\circ}$ using the $\mathrm{x}$-wire probe appear in Figure 8 , and indicate that the vortex is stronger than at $\alpha=4.5^{\circ}$, with tangential velocities of the order of $10 \%$ of the freestream velocity.

Contours of the axial (streamwise) mean velocity, as measured with the $\mathrm{x}$-wire probe, are illustrated in Figures 9 and 10 for the model incidences of $4.5^{\circ}$ and $13^{\circ}$, respectively. The five-hole probe results are not shown, as they are not very descriptive of core axial velocity due to a lack of measurement points close to the vortex axis. For $\alpha=4.5^{\circ}$, Figure 9 reveals an axial velocity deficit in the core of the tip vortex, where the ratio of axial velocity to freestream velocity is about $U / U_{\infty}=0.9$. Figure 10 indicates an even stronger axial velocity deficit at $\alpha=13^{\circ}\left(U / U_{\infty}=0.7\right)$ than at $\alpha=4.5^{\circ}$. Furthermore, at $\alpha=13^{\circ}$, the region of axial velocity deficit is more than twice as large as at $\alpha=4.5^{\circ}$, and a secondary region of deficit, located outboard and slightly above the main region, appears.

The wing and wing-tip geometry in the present study is more complex than in most previous tip vortex studies, and has not been investigated before now. However, the appearance of an axial velocity deficit in the tip-vortex core, immediately downstream of the wing trailing edge, was reported in past studies on trailing vortices generated by rectangular half-wings (e.g. Shekarriz et $a l^{8}{ }^{8}$ ). Nevertheless, there exists considerable disparity in the literature. For example, some authors observed an axial velocity surplus which reached a maximum near the wing trailing edge $\left(1.4 U_{\infty}\right.$ by Chigier and Corsiglia ${ }^{9}$ and $1.77 U_{\infty}$ by Chow et al. ${ }^{3}$ ), and then diminished, usually becoming a deficit a few chordlengths downstream. Still others have shown an axial velocity surplus persisting further downstream (e.g. Green and $\mathrm{Acosta}^{10}$ ). These variations are very likely due to the differences in wing and wing-tip geometry (eg. wing profile, aspect ratio and tip shape) between the cited studies.

In the present study, the investigated flow is in fact the combination of the tip vortex of the F/A-18 wing and the wake of the AIM-9 tip missile. In addition, the wake of the main body of the tip missile, which can be compared to that of an ogive cylinder, is further complicated by interactions with the wakes of the missile fins and canards. It is clear that the velocity deficit due to the tip missile body contributes to the axial velocity deficit in the central region of the tip vortex, and more so as the model angle of attack increases. The tip missile, whose influence on the flow thus increases with $\alpha$, is also likely to be responsible for the secondary region of axial velocity deficit which appears at $\alpha=13^{\circ}$. 


\section{Reynolds Stresses and Turbulent Kinetic Energy in the Plane $x / c=4.2$}

The normal components of the Reynolds stress tensor, non-dimensionalized by the freestream velocity $\left(U_{\infty}=244 \mathrm{~m} / \mathrm{s}\right)$, are shown in Figures 11 and 12 for the model incidences of $4.5^{\circ}$ and $13^{\circ}$, respectively. These stresses were obtained from the $\mathrm{x}$-wire probe measurements. Each $\mathrm{x}$-wire measurement was repeated with the probe rotated by $90^{\circ}$ about its axis in order to obtain all three velocity components.

At both angles of attack, the turbulence levels of all the components were highest in the core region of the tip vortex, where the streamwise velocity deficit had been measured. At $\alpha=4.5^{\circ}$, the maximum transverse stresses, $\overline{v^{\prime 2}}$ and $\overline{w^{\prime 2}}$, were slightly higher than, but of the same order of magnitude as, the maximum streamwise stress, $\overline{u^{\prime 2}}$ (Figure 11). Specifically, the highest levels of the streamwise, vertical and lateral normal stresses, normalized by the freestream velocity, were measured to be $1.1 \times 10^{-3}, 1.4 \times 10^{-3}$ and $1.5 \times 10^{-3}$, respectively. At $\alpha=13^{\circ}$, the normal stresses were an order of magnitude higher than those measured at $\alpha=4.5^{\circ}$ : the maximum normalized streamwise, vertical and transverse stresses were 0.019 , 0.014 and 0.024 , respectively (Figure 12). Also, the core region of maximum stresses was centered further inboard, and occupied a greater area, than at the lower model incidence. The secondary structure slightly above and outboard of the main vortex, which was previously identified in the streamwise mean velocity contours, is visible in the plots of all three components of the Reynolds normal stress at $\alpha=13^{\circ}$.

The distribution of the Reynolds shear stresses, $\overline{u^{\prime} v^{\prime}}$ and $\overline{u^{\prime} w^{\prime}}$, as measured with the $\mathrm{x}$-wire probe in the plane $x / c=4.2$, are shown in Figures 13a and $\mathrm{b}$ for $\alpha=4.5^{\circ}$ and in Figures $14 \mathrm{a}$ and $\mathrm{b}$ for $\alpha=13^{\circ}$. The measurements obtained at $\alpha=4.5^{\circ}$ indicate that both of these stresses are negative everywhere in the wingtip region. The shear stress $\overline{u^{\prime} v^{\prime}}$ reaches a local minimum of $-7.3 \times 10^{-5} U_{\infty}{ }^{2}$ in the vortex core region, while the minimum value of $\overline{u^{\prime} w^{\prime}}\left(-4.0 \times 10^{-5} U_{\infty}^{2}\right)$ is found slightly outboard of the vortex core. At $\alpha=13^{\circ}$, the overall peak (absolute) levels of both $\overline{u^{\prime} v^{\prime}}$ and $\overline{u^{\prime} w^{\prime}}$ were $2.2 \times 10^{-3} U_{\infty}^{2}$, which is two orders of magnitude higher than at the lower angle of attack. Typically, the shear stresses $\overline{u^{\prime} v^{\prime}}$ and $\overline{u^{\prime} w^{\prime}}$ possess both positive and negative lobes when plotted in the cross-stream plane of a wing-tip vortex (cf. Chow et al. ${ }^{3}$ ). These lobes are not visible in the results obtained at $\alpha=4.5^{\circ}$, as the magnitude of the measured stresses is likely too low (less than $7.5 \times 10^{-5} U_{\infty}{ }^{2}$ ) to be resolved accurately with the hot-wire anemometer. However, the two-lobe pattern can be observed for $\alpha=13^{\circ}$, and is most easily discernable when the shear stresses are plotted in the form of dimensionless correlation coefficients (shear stress non-dimensionalized by the corresponding root mean-squared velocities) as shown in Figure 15. In Figure 15, the lobes are aligned horizontally for $\overline{u^{\prime} v^{\prime}}$, and vertically for $\overline{u^{\prime} w^{\prime}}$. Peak levels of the shear stress correlation coefficients were in the range 0.3 to 0.5 , which is typical of other turbulent shear flows such as wakes and boundary layers.

Contours of the turbulent kinetic energy, $\overline{k^{2}}=\overline{u^{\prime 2}}+\overline{v^{\prime 2}}+\overline{w^{\prime 2}}$, are plotted in Figures 16 and 17 for $\alpha=4.5^{\circ}$ and $\alpha=13^{\circ}$, respectively. As expected, the kinetic energy is highest in the core region, and diminishes with radial distance outwards. At $\alpha=13^{\circ}$, the maximum turbulent energy is an order of magnitude higher, and the region of highly turbulent flow is larger, than at $\alpha=4.5^{\circ}$. The secondary structure identified in the plots of axial velocity and normal stresses is clearly visible in Figure 17, and is likely due to the wake of the tip missile. The main body of the missile, like an ogive cylinder, produces a larger wake with higher turbulence levels at greater incidence. In addition, the wakes of the canards and fins on the missile introduce additional vorticity and turbulence into the flow downstream of the wing-tip.

\section{Conclusions}


The investigation of the wing-tip vortex generated by a model F/A-18 fitted with AIM-9 tip missiles, via five-hole probe measurements as well as hot-wire anemometry, has provided an overview of the mean and turbulent flowfield. At $\alpha=4.5^{\circ}$, the tip vortex showed certain similarities with some of those described in the literature and generated at lower speeds with wings of simpler geometry. At $\alpha=13^{\circ}$, the vortex increased in strength and size, the turbulence levels were an order of magnitude higher than at $\alpha=4.5^{\circ}$, and the effects of the tip missile on the flow were more prominent. The missile body produced an axial velocity deficit which became more significant at the higher angle of attack, as well as increased turbulence levels due to its own wake and that of its canards and fins. The tip missile thus significantly increases the complexity of the studied flow. More detailed measurements, ideally at several streamwise stations, could help to understand more fully the development of this complex flowfield.

\section{References}

1 DEVENPORT, W.J., RIFE, M.C., LiAPIS, S.I. \& Follin, G.J.: The structure and development of a wingtip vortex. J. Fluid Mech. 312 (1996), 67--106.

2 DEVEnPORT, W.J., Zsoldos, J.S. \& Vogel, C.M.: The structure and development of a counterrotating wing-tip vortex pair. J. Fluid Mech. 332 (1997), 71--104.

3 CHOW, J.S., ZilliaC, G.G. \& BRADSHAW, P.: Mean and Turbulence Measurements in the Near Field of a Wingtip Vortex. AIAA J. 35, No. 10 (1997), 1561--1567.

4 Ohman, L.H., Brown, D., Chan, Y.Y., Galway, R.D., Hashim, S.M., Khalid, M., MaleK, A., MOKRY, M., TANG, N. \& THAIN, J.: New Transonic Test Sections for the NAE 5FTx5FT Trisonic Wind Tunnel. NAE-AN-62, National Research Council Canada (1990).

5 Ohman, L.H., Brown, D.: Performance of the New Roll-in Roll-out Transonic Test Section of the NAE $1.5 \mathrm{~m} \times 1.5 \mathrm{~m}$ Blowdown Wind Tunnel. Proceedings of the $17^{\text {th }}$ Congress of the International Council of the Aeronautical Science, Stockholm, Sweden (1990), 363--387.

6 OHMAN, L.H.: Calibration of the 5-Hole Probe Flow Survey Rakes in the Lockheed Compressible Flow Wind Tunnel. DHC-ISTC 92-16 (1992).

7 TANG, F.C., LeE, B.H.K., Ohman, L.H. \& JiAng, L.Y.: Flowfield Studies Around a CF-18 Wing at Moderate Angle of Attack. 44 CASI Conference, Toronto (1997).

8 SheKARRIZ, A., FU, T.C., KATZ, J. \& HuAnG, T.T.: Near-Field Behavior of a Tip Vortex. AIAA J. 31, No. 1 (1993), 112--118.

9 Chigier, N.A. \& CoRsiglia, V.R.: Tip Vortices - Velocity Distributions. NASA TM X-62, 087 (1971).

10 GreEN, S.I. \& ACOSTA, A.J.: Unsteady flow in trailing vortices. J. Fluid Mech. 227 (1991) 107--134. 


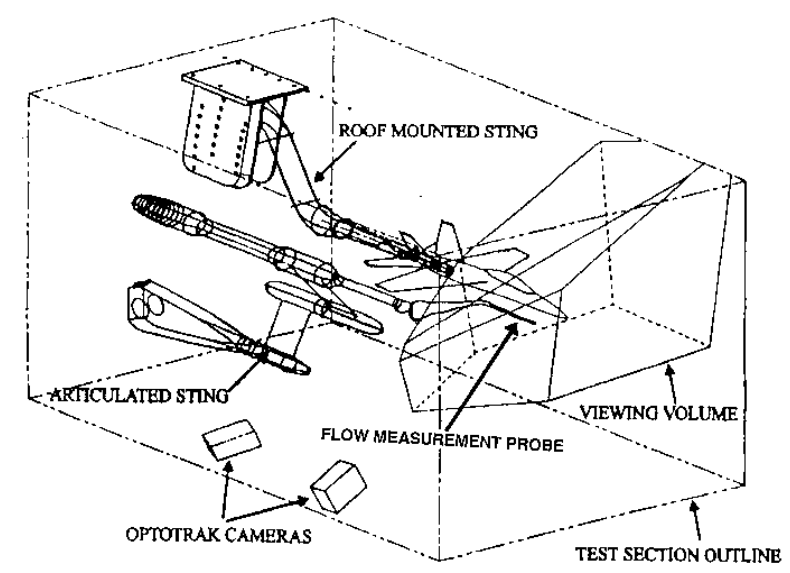

Figure 1 Wind tunnel setup for flowfield survey around $6 \%$ scale F/A-18 model.

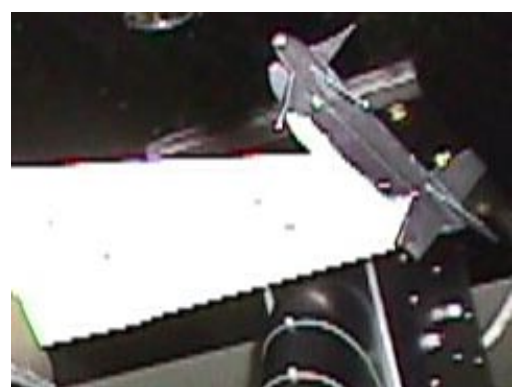

Figure 2 AIM-9 sidewinder missile mounted on the model F/A-18 wing-tip.

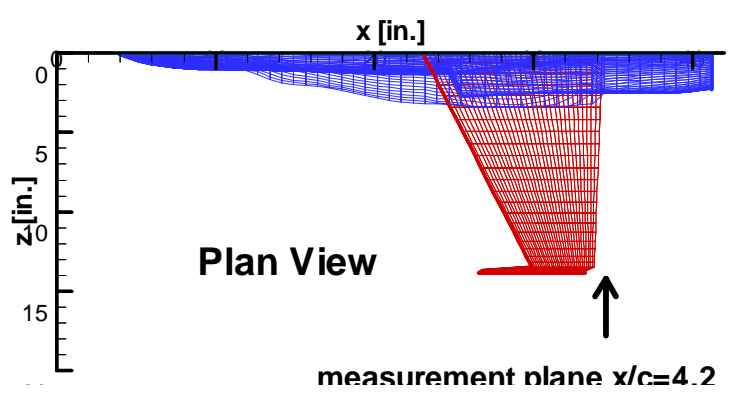

Figure 3 Reference coordinate system for flowfield survey.

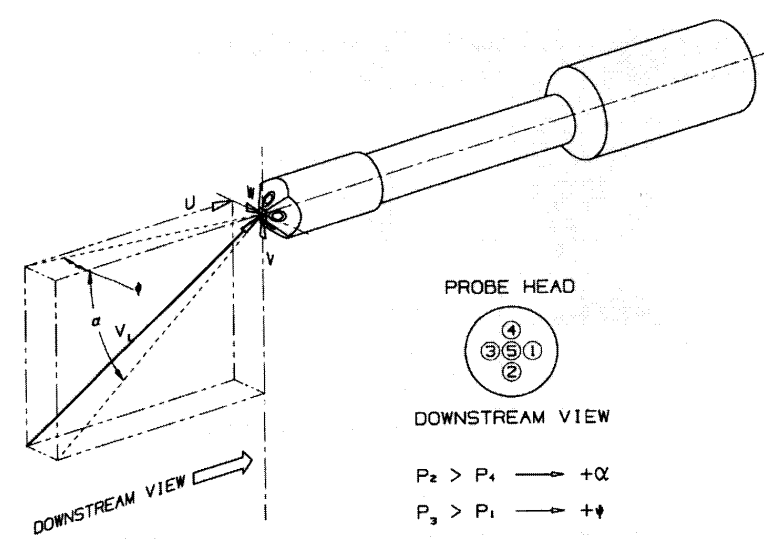

Figure 4 Schematic of five-hole probe.
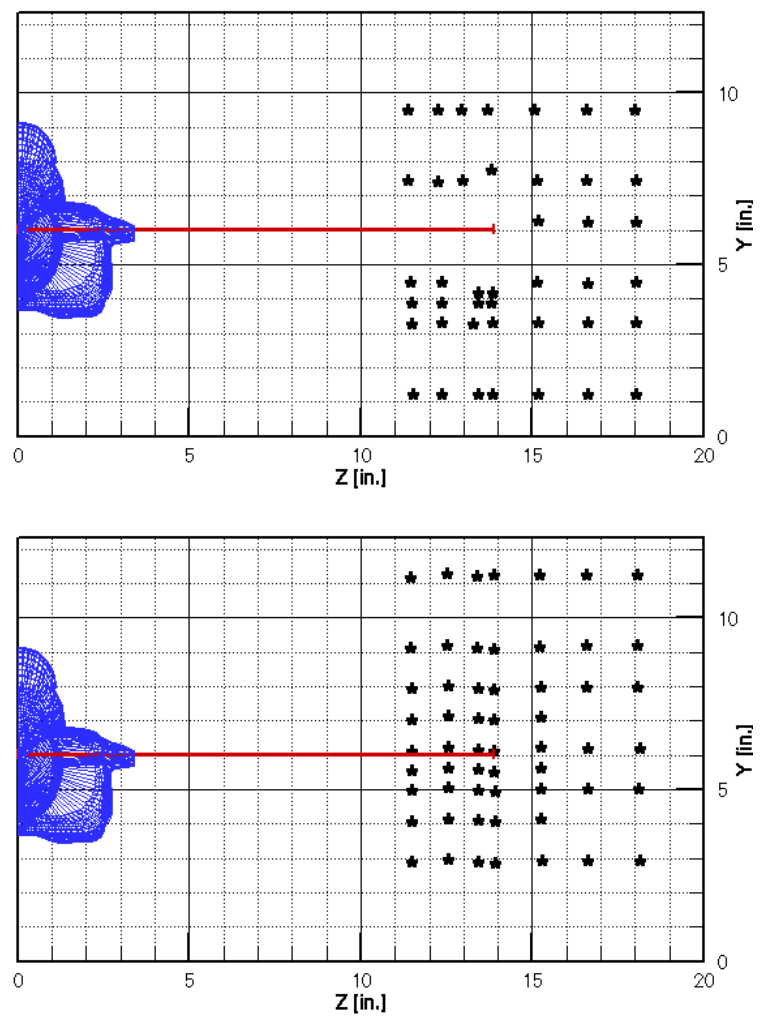

Figure 5 Grid for five-hole probe (top) and hot-wire (bottom) measurements in the plane $x / c=4.2$. 


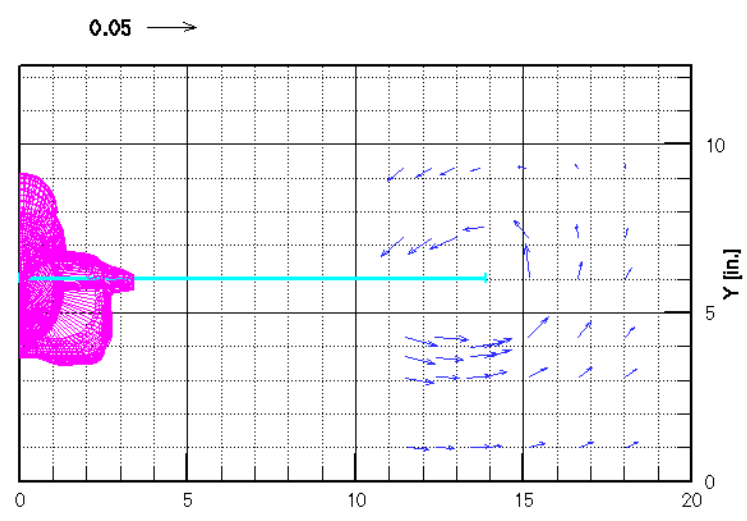

Figure 6 Cross-flow velocity vectors, normalized by the freestream velocity, in the plane $x / c=4.2$, at $\alpha=4.5^{\circ}$, as measured with the five-hole probe.

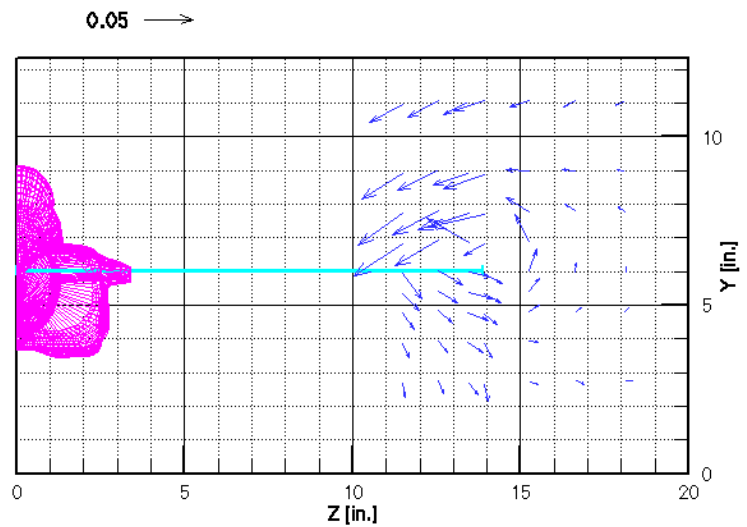

Figure 7 Cross-flow velocity vectors, normalized by the freestream velocity, in the plane $x / c=4.2$, at $\alpha=4.5^{\circ}$, as measured with the $\mathrm{x}$-wire probe.

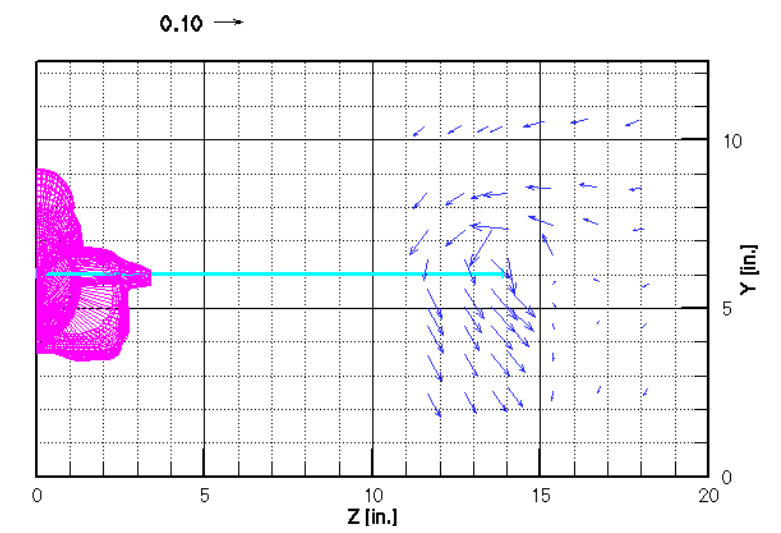

Figure 8 Cross-flow velocity vectors, normalized by the freestream velocity, in the plane $x / c=4.2$, at $\alpha=13^{\circ}$, as measured with the $\mathrm{x}$-wire probe.

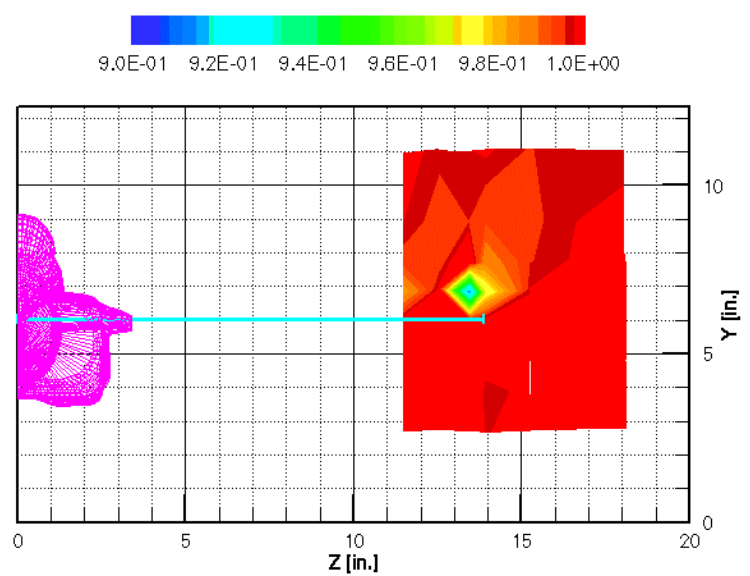

Figure 9 Normalized axial mean velocity contours in the plane $x / c=4.2$, at $\alpha=4.5^{\circ}$, as measured with the $x$-wire probe.

$\begin{array}{llllll}7.0 \mathrm{E}-0 & 7.6 \mathrm{E}-01 & 8.2 \mathrm{E}-01 & 8.8 \mathrm{E}-01 & 9.4 \mathrm{E}-01 & 1.0 \mathrm{E}+00\end{array}$

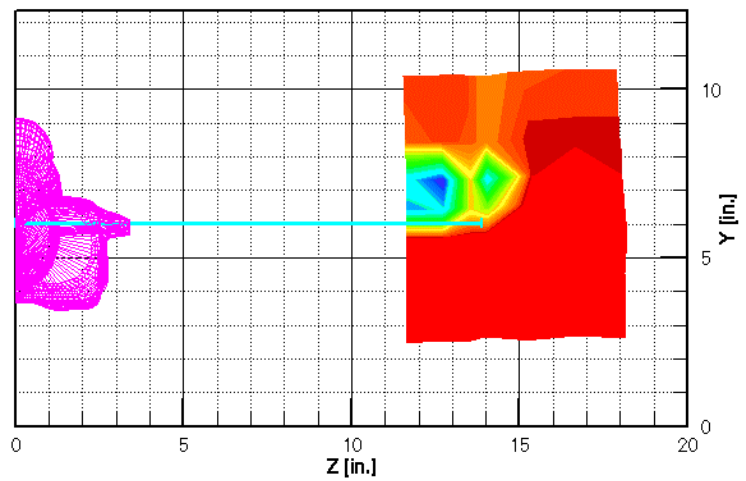

Figure 10 Normalized axial mean velocity contours in the plane $x / c=4.2$, at $\alpha=13^{\circ}$, as measured with the $x$-wire probe. 

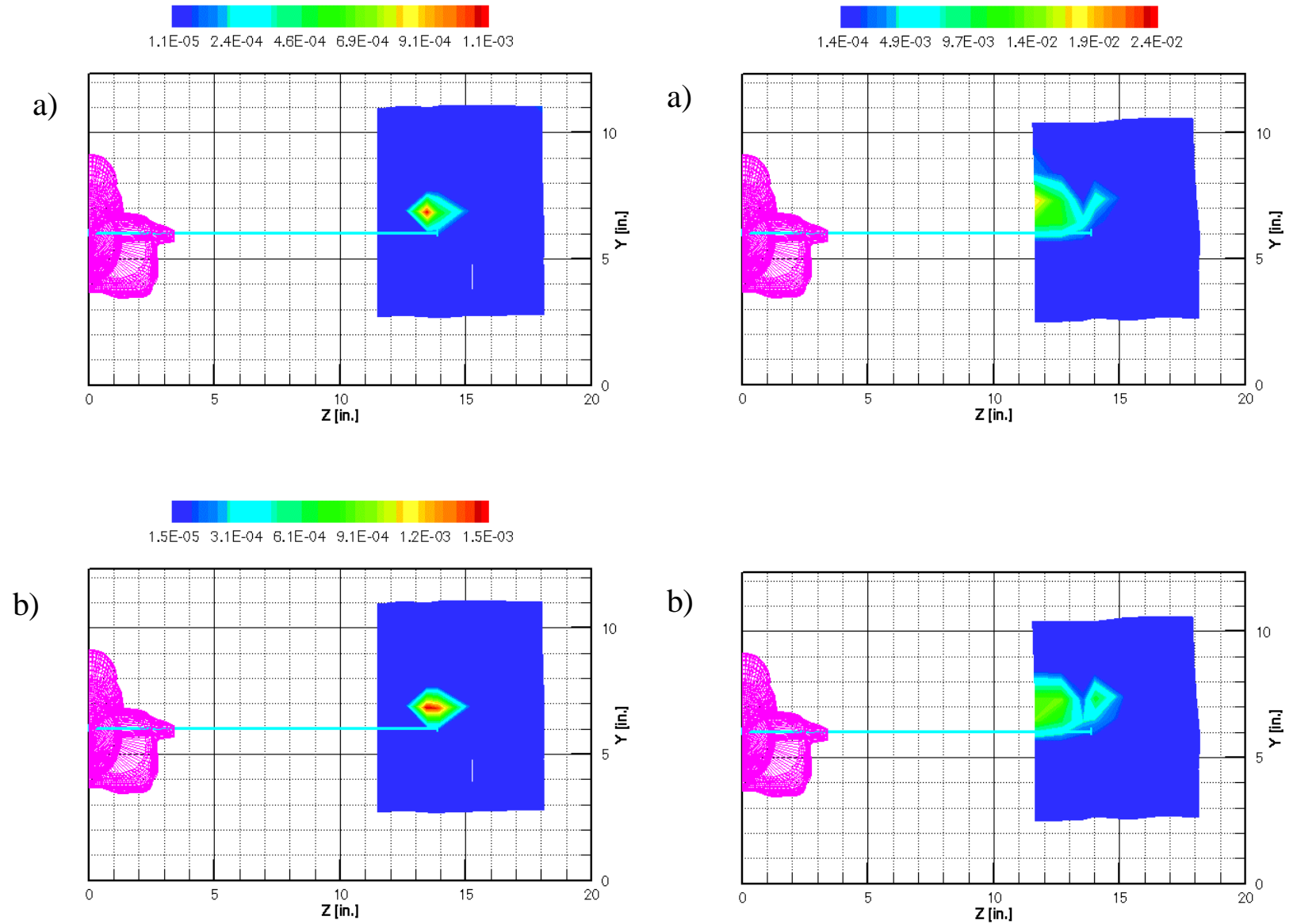

b)

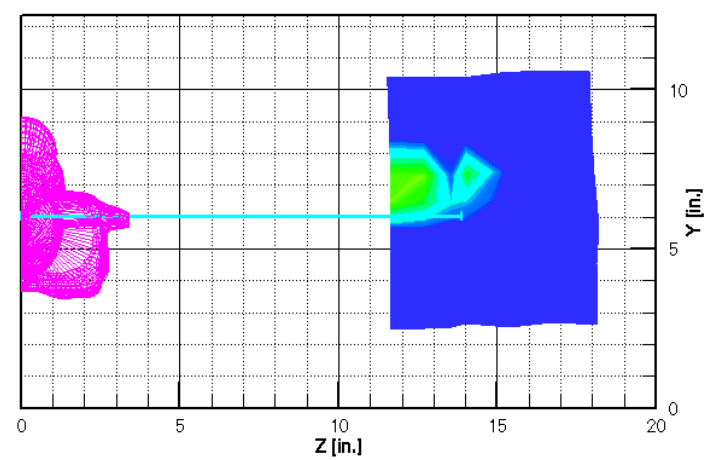

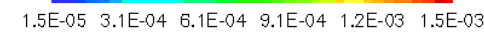

c)

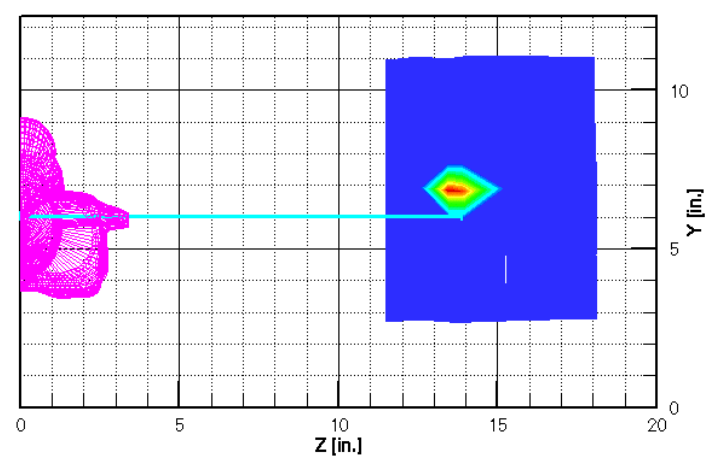

Figure 11 Contours of the normal components of the Reynolds stress tensor (normalized by the freestream velocity) at $x / c=4.2, \alpha=4.5^{\circ}$, as measured with the $\mathrm{x}$-wire probe. a) $\overline{u^{\prime 2}} / U_{\infty}^{2}$; b) $\overline{v^{\prime 2}} / U_{\infty}^{2}$; c) $\overline{w^{\prime 2}} / U_{\infty}^{2}$. c)

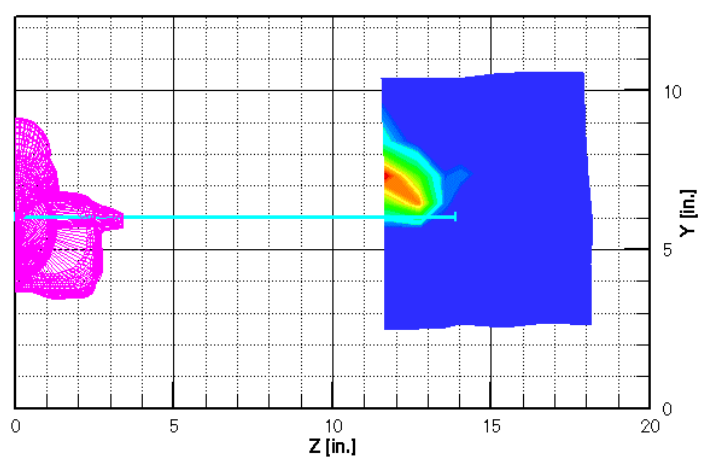

Figure 12 Contours of the normal components of the Reynolds stress tensor (normalized by the freestream velocity) at $x / c=4.2, \alpha=13^{\circ}$, as measured with the $\mathrm{x}$-wire probe. a) $\overline{u^{\prime 2}} / U_{\infty}^{2}$; b) $\overline{v^{\prime 2}} / U_{\infty}^{2}$; c) $\overline{w^{\prime 2}} / U_{\infty}^{2}$. 


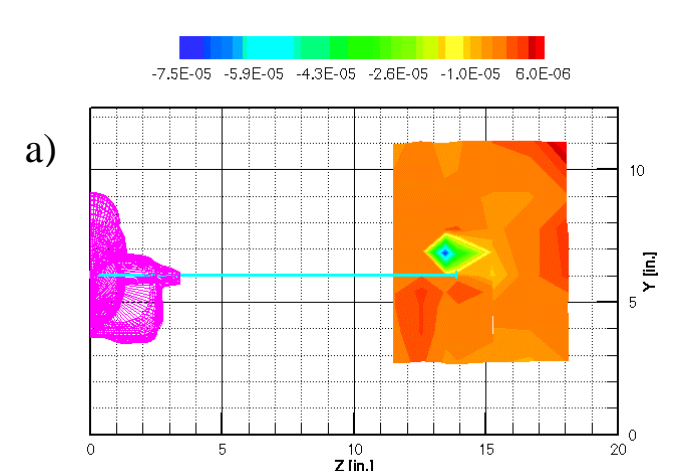

b)

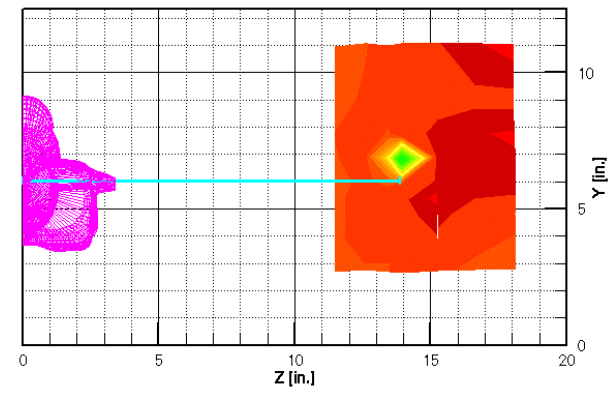

Figure 13 Contours of the normalized Reynolds shear stresses at $x / c=4.2, \alpha=4.5^{\circ}$. a) $\overline{u^{\prime} v^{\prime}} / U_{\infty}^{2}$; b) $\overline{u^{\prime} w^{\prime}} / U_{\infty}^{2}$.

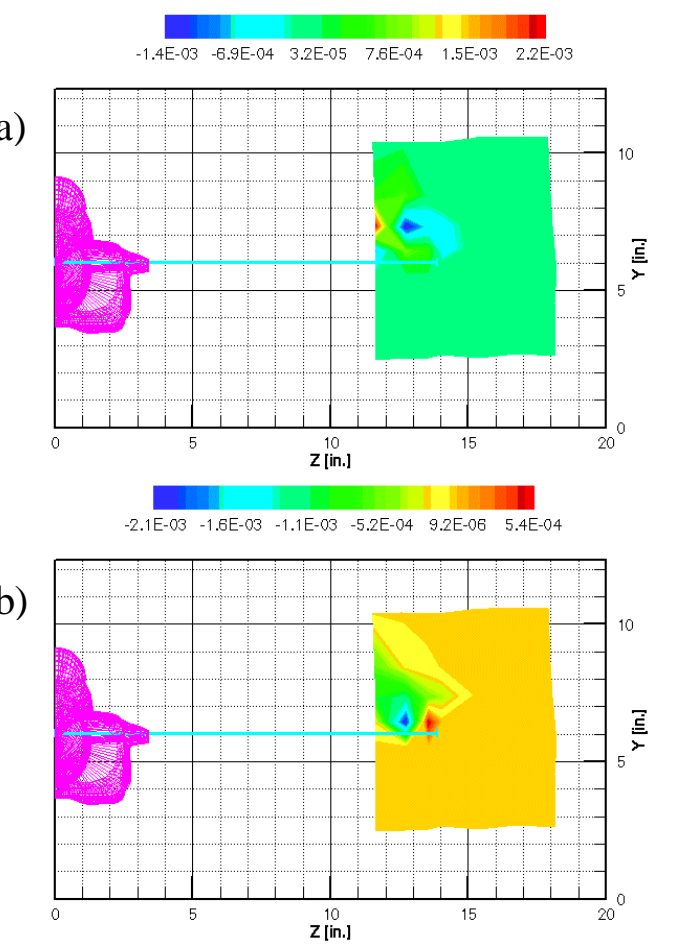

Figure 14 Contours of the normalized Reynolds shear stresses at $x / c=4.2, \alpha=13^{\circ}$. a) $\overline{u^{\prime} v^{\prime}} / U_{\infty}^{2}$; b) $\overline{u^{\prime} w^{\prime}} / U_{\infty}^{2}$. a)

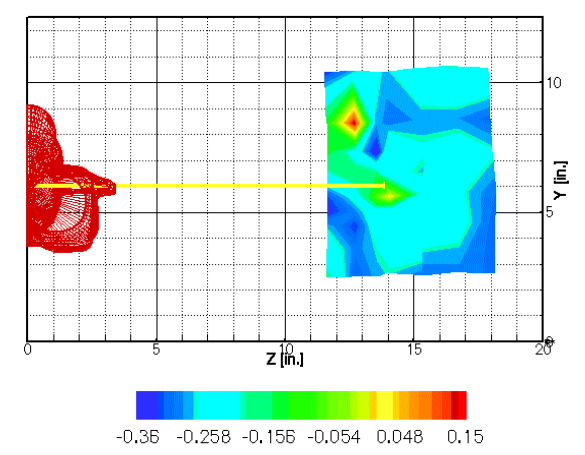

b)

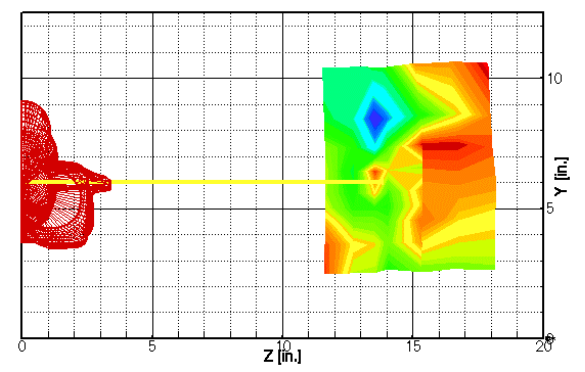

Figure 15 Contours of the Reynolds shear stress correlation coefficients at $x / c=4.2, \quad \alpha=13^{\circ}$. a) $\frac{\overline{u^{\prime} v^{\prime}}}{\sqrt{\overline{u^{\prime 2}}} \sqrt{\overline{v^{\prime 2}}}}$; b) $\frac{\overline{u^{\prime} w^{\prime}}}{\sqrt{\overline{u^{\prime 2}}} \sqrt{\overline{w^{\prime 2}}}}$.

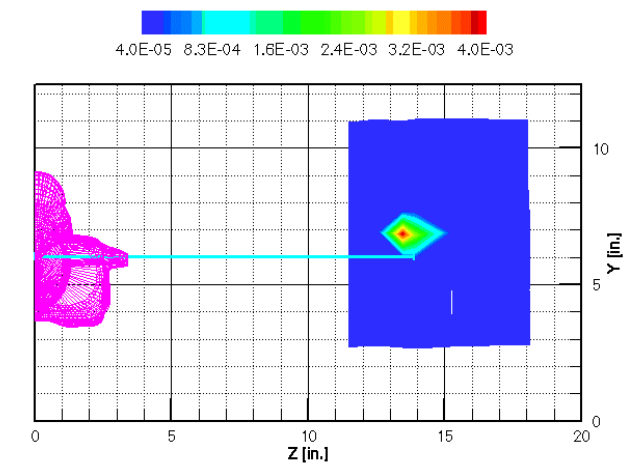

Figure 16 Contours of the normalized turbulent kinetic energy $\overline{k^{2}} / U_{\infty}^{2}$ at $x / c=4.2, \alpha=4.5^{\circ}$.

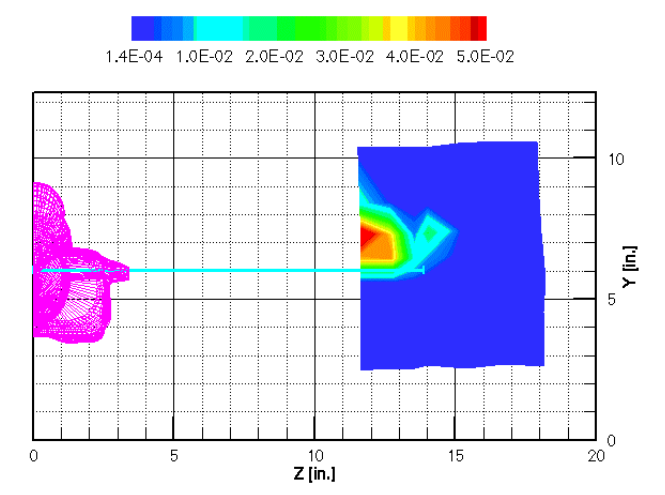

Figure 17 Contours of the normalized turbulent kinetic energy $\overline{k^{2}} / U_{\infty}^{2}$ at $x / c=4.2, \alpha=13^{\circ}$. 